\title{
Preprint
}

Bernard, P., St-Amour, S., Lachance, Kingsbury, C., \& Lapointe. (2020). Dynamic patterns of depressive symptoms and sleep during the first month of strict lockdown in two women with major depressive disorder. PsyArXiv. https://doi.org/10.31234/osf.io/5enrq

Dynamic patterns of depressive symptoms and sleep during the first month of COVID strict lockdown in two women with major depressive disorder

\section{Paquito BERNARD ${ }^{1,2^{*}}$, Samuel ST-AMOUR ${ }^{1,2,3}$, Jean-Philippe LACHANCE ${ }^{2,3}$, Celia KINGSBURY ${ }^{1,2}$, Josyanne LAPOINTE ${ }^{1,2}$}

${ }^{1}$ Department of Physical Activity Sciences, Université du Québec à Montréal, Montréal, Québec, Canada

${ }^{2}$ Research Center of the Montreal Mental Health University Institute, Montreal, Quebec, Canada ${ }^{3}$ Health and Society Institute, Université du Québec à Montréal, Montreal, Canada

*Correspondence concerning this article should be addressed to:

Paquito Bernard, PhD, Université du Québec à Montréal, Montréal, Canada Faculté des sciences, Avenue du Président Kennedy, Montréal, Québec, Canada, H2X 1Y4 bernard.paquito@uqam.ca,@,PaquitoBernard ORCID: https://orcid.org/0000-0003-2180-9135 


\begin{abstract}
This article aimed to document the day-to-day patterns of depressive symptoms and sleep parameters, and to explore the dynamic network structure of depressive symptoms during the first COVID strict lockdown. Two participants with major depressive disorder were included in a 30days observational ecological momentary assessment study just before the lockdown in Montreal, Canada. In both cases, the self-reported depressive symptoms and core affects fluctuated during the lockdown. However, all depressive symptoms were not systematically exacerbated. Among them, significant linear and non-linear temporal patterns have been identified. For case 1, the lockdown period did not worsen the sleep quality and sleep parameters. The psychomotor retardation, fatigue, appetite loss and level of arousal played a prominent role in the idiographic dynamic symptom networks. These case studies allow a granular understanding of the lockdown effects on depressive symptoms and affective experiences dynamics, and highlight the need for person-centered mental health care to help people with major depressive disorder.
\end{abstract}

Key words: idiographic, depression, sleep efficiency, COVID-19, network 


\section{Introduction}

Experts suggested that the COVID-19 pandemic may be challenging for adults with mental disorders due to disruptions in treatment and loneliness (Holmes et al., 2020). Higher scores of depression, anxiety and sleep disorders were reported in Chinese adults with severe mental disorders during the COVID-19 lockdown in an online cross-sectional study (Hao et al., 2020). More than fifty percent of German adults with mental disorders suffer higher mental distress due to COVID-19 (Frank et al., 2020). Impaired sleep was observed in a quarter of the patients with affective disorders. A study did not observe a symptom's exacerbation in adults with major depressive disorders pre- and post-lockdown due to COVID-19. However, the post-measures were collected 2 to 4 months after the lockdown. Authors suggested that an aggravation of symptoms may appear during the first weeks post lockdown, and then "improvement" could be noted, i.e., a potential non-linear pattern (Pinkhlma et al., 2020).

The mental health research community needs to collect high-quality data in order to understand the effects of the COVID-19 pandemic and the associated lockdown measures in adults with mental disorders (Holmes et al., 2020). In this perspective, studies with real time monitoring methods (also known as ecological momentary assessments; EMA) contribute refining the understanding of depressive symptoms dynamic during a specific period (Wichers, 2014). Indeed, EMAs enable the characterization of dynamic patterns within (e.g., variability, inertia, instability) and between core symptoms (e.g., networks). A growing number of psychopathology investigations showed that these proprieties are relevant in the etiology and prediction of major depressive disorders (Borsboom \& Cramer, 2013; Marieke Wichers et al., 2016).

Several experts suggested that lockdown may have detrimental effects on population mental health, with worst effects in adults with preexisting mental health disorders (Pfefferbaum \& North, 2020; Taquet et al., 2021). Thus, an EMA study compared symptoms levels pre- and during pandemic 
self-reported symptoms, substance use, well-being and total sleep time in adults with affective disorders in three US states (Pinkham et al., 2020). The mood and psychotic symptoms were not exacerbated during the first COVID-19 wave. A significant increase in well-being and substance use was found. This suggests that higher substance use could be a coping strategy for participants during the pandemic's early months. However, this study presents three methodological weaknesses diminishing the results' validity: participants were not necessarily included during a local lockdown, EMAs were carried out during a short period (9 days), and assessed symptoms were not specific to major depressive disorders (Pinkham et al., 2020).

An observational EMA study including adults with major depressive disorders was ongoing in our laboratory since April 2018. This study was initially designed to model the bidirectional daily associations between depressive symptoms and sleep parameters during 30 consecutive days and identify clinical subtypes of depression. Two participants were included just before the strict lockdown in Montreal, Canada. By adopting an idiographic approach, we aimed to document the day-to-day patterns of depressive symptoms and sleep parameters, and explore the dynamic network structure of measured depressive symptoms during the first four weeks of strict lockdown. The second aim is based on the network theory of mental disorders, i.e., the mental disorders arise from the causal interaction between symptoms in a network (Borsboom, 2017).

\section{Methods}

\section{Participants}

Participants were recruited from Université du Québec à Montréal through online advertisements. Interested participants were screened using the Center for Epidemiologic Studies Depression scale (CES-D) during a brief phone call. A participant was included when his total score was higher than 18 (Morin, Moullec, et al., 2011).

Participants were then invited to attend an in-person structured clinical interview. The Stuctured Clinical Interview for Diagnostic and Statistical Manual of Mental Disorder-5 (SCID-5) was 
administered by an experienced $\mathrm{PhD}$ student in clinical psychology. Inclusion criteria were a primary diagnosis of major depressive disorder and 20-55 years of age. Exclusion criteria were pregnancy, functional limitations, and any history of bipolar disorder, psychosis or substance use disorders (except for tobacco).

\section{Measures}

\section{Background measures}

The severity of depressive symptoms was assessed using CES-D. This 21-item self-report questionnaire measured the following depressive symptoms: positive affect, depressive affect, somatic complaints, and disturbed interpersonal relationships. Scores range is from 0 to 60 , with higher scores indicating greater depressive symptoms (Morin, Moullec, et al., 2011). The Beck Anxiety Inventory (Muntingh et al., 2011) was used to evaluate the anxiety severity (21 items, 4point Likert responses). The total score range is from 0 to 63. Alcohol Use Disorders Identification Test short form (Bradley et al., 2007) was used to assess alcohol misuse. Insomnia severity was measured with Insomnia Severity Index (Morin, Belleville, et al., 2011). It is a 7-item questionnaire. A 5-point scale is used to rate each item, yielding a total score ranging from 0 to 28 . A higher score indicates higher insomnia symptoms.

\section{Objective sleep measures}

Sleep was measured with a validated GENACTIV accelerometer (van Hees et al., 2018). Participants were asked to wear this accelerometer on their non-dominant arm's wrist. Data were not visible to participants while they are wearing the device. Nocturnal actigraphic data (van Hees et al., 2018) were analyzed with "ggir" R package to obtain the following sleep parameters: sleep efficiency (i.e., total sleep time divided by total time in bed), and total sleep time (i.e., time in bed minus total wake time). 


\section{Daily assessments}

A research-oriented app was installed (EthicaData, https://ethicadata.com/) on participants' smartphones. All items were self-measured three times per day, except for sleep quality (morning only). Participants reported their depressive symptoms three times a day (app on smartphone), and sleep parameters were monitored for 30 days with a validated accelerometer (GENACTIV). For each time point, participants rated the severity of the following symptoms using a $0-100$ visual analog slider (0: not at all - 100: as much as possible): fatigue, guilt, diminished concentration, loss of appetite, psychomotor retardation, and sleep quality. These items were previously used in EMA studies (Moullec et al., 2011). The core affects (i.e., valence and level of arousal) were measured with six items from the Swedish short self-report measure of core affect (Västfjäll \& Gärling, 2007) using bipolar adjectives scales. All EMA items are presented in Table 1.

\section{Statistical analyses}

Temporal patterns of depressive symptoms and sleep parameters were modeled using Generalized Additive Models (Wood, 2017). This nonparametric model allows non-linear relationships to be flexibly modeled without specifying the functional form. Two graphical vector autoregressive models (graphicalVAR package) were carried out to estimate the idiographic structure of depressive symptoms (Epskamp et al., 2018). Contemporaneous and temporal networks were obtained for each participant. The nodes and edges represent the variables and the associations between variables, respectively. In the idiographic temporal network, the edges indicate which symptom predicts other symptoms in the next time point. In the idiographic contemporaneous network, the edges represent associations between the symptoms, after controlling for all the other symptoms in the previous- and same timepoint (Borsboom, 2017).

These networks allowed to identify the most critical symptoms and symptom variations within individuals and across time. As recommended for the relatively short EMA time series (Beck \& Jackson, 2020), we used the Bayesian information criterion to optimize prediction accuracy. Data 
preparation and statistical analyses were performed with R 4 and the following packages were used: mgcv, bootnet, visreg, ggir, and lubridate (Epskamp et al., 2018; Wood, 2017). The R code and data for this study are available here: https://osf.io/9a8s5/.

\section{Results}

Descriptive statistics and case characteristics are provided in Table 1.

\section{Case 1}

Case 1 was a 36-year-old female with a history of multiple episodes of major depressive disorder with antidepressant use (tetracyclic), and a short total sleep time (daily mean $=5.2$ hours). She began to work from home one week after the lockdown. Her loss of appetite significantly linearly increased overall but a decrease was observed within the last 5 days of the study $(P=$ 0.002, $\mathrm{R}^{2}=10 \%$ ), see Supplementary file for details (https://osf.io/9a8s5/). A significant decrease of negative affects and level of arousal was observed during the first three days of lockdown, then they stabilized at more "neutral" levels $\left(P<0.006, \mathrm{R}^{2}=22 \%\right.$; see details in Figure 1). No significant exacerbation of other depressive symptoms and objectively assessed sleep parameters were found. The psychomotor retardation, fatigue symptoms, appetite loss and level of arousal (item 3) were the most influential in contemporaneous networks (see Figure 2). In the temporal network, psychomotor retardation and level of arousal (item 1) had the highest outdegree estimates, indicating that their levels had the strongest influence on symptoms at $\mathrm{T}+1$. Based on indegree indices, the affective valence (item 3) was the symptom the most influenced by variability of symptom at T-1 (Figure 2). The centrality plots are provided in the supplementary file.

\section{Case 2}

Case 2 was a 20-year-old unemployed female recruited during her second major depressive disorder episode and treated with sertraline. She was unemployed during the lockdown. A significant linear increase of psychomotor retardation and fatigue was found $\left(P=0.005, \mathrm{R}^{2}=5 \%\right)$. A significant non-linear pattern was observed for the third arousal item (awake - sleepy). A negative 
affect (pleased - displeased) significantly increased during the first eight days, then slowly decreased until baseline level $\left(P<0.007, \mathrm{R}^{2}=8 \%\right)$. Total sleep time was significantly improved during the first five days, and then stayed above 7.5 hours for the next nights. The strength indices indicated negative affect nodes were interdependent, and were associated with lower psychomotor retardation and fatigue level, respectively in the contemporaneous network. Negative affect nodes were interdependent, and were associated with lower psychomotor retardation and fatigue level, respectively. For temporal network, guilty, affective valence (items 2 and 3) had the highest degree outdegree estimates, indicating that their levels had the strongest influence on symptoms at $\mathrm{T}+1$. Indegree indices suggest that affective valence 3 was the most influenced symptom by variability of symptoms at T-1 (Figure 3). More details about models and plots are available in Supplementary file.

\section{Discussion}

In both cases, self-reported depressive symptoms and core affects fluctuated during the lockdown. However, all measured depressive symptoms were not systematically exacerbated. Among them, significant linear and non-linear temporal patterns have been identified. These findings provide a more granular perspective of depressive symptoms during a lockdown in comparison to previous cross-sectional studies (Franck et al., 2020, Hao et al., 2020). Furthermore, the non-linear patterns of depressive symptoms may explain the null findings of Pinkhlma et al. (2020).

Another important finding was that poor sleep quality and deteriorated sleep parameters (for case 1) were not worsened during the lockdown. Psychomotor retardation and level of arousal played a prominent role in the idiographic dynamic symptom networks. Networks findings allowed the identification of the most influential symptoms for each participant (David et al., 2018; Fisher et al., 2017). The symptoms with the highest strength indices described in the findings may be considered as an activation symptoms of networks, i.e., networks' activation triggers a self-sustaining network 
of symptoms (Borsboom 2017). These may be targeted to personalize a psychological treatment post-lockdown, as suggested by Fisher et al. 2017. Two types of psychological interventions could be carried out: symptom interventions (i.e., directly change the state of one or more symptoms; see for instance Fisher et al. 2019), or network interventions (i.e., "change the network structure itself by modifying symptom-symptom connections"; see an example with Snippe et al. 2017).

This is the first study to examine depressive symptoms during the first four weeks of lockdown. The main strength of this study is its ecological validity since participants were assessed in their environment, minimizing the risk of recall bias (Wichers, 2014). Additionally, sleep parameters were objectively assessed. This is particularly relevant because sleep studies have shown weak correlations between self-reported and objective measures in poor sleepers (Roth et al., 2006). Nonetheless, this study has some limitations. Only depressive symptoms were measured, although a previous investigation found that anxiety symptoms were highly prevalent in adults with major depressive disorders and may play a central role in symptom networks (Fisher et al. 2017). Moreover, despite their clinical relevance, suicidality and diminished interest in activities symptoms were not included in our EMAs (Maj et al., 2020).

These case studies contribute to our understanding of the lockdown effects on depressive symptoms and affective experiences dynamics, and highlight the need for person-centered mental health care to help people with major depressive disorder during a lockdown. Practically speaking, the symptoms with the most preponderant impact on other symptoms within the idiosyncratic network could be considered as priority treatment targets in cognitive-behavioral therapy. 


\section{References}

Beck, E. D., \& Jackson, J. J. (2020). Consistency and change in idiographic personality: A longitudinal ESM network study. Journal of Personality and Social Psychology, 118(5), 1080-1100. https://doi.org/10.1037/pspp0000249

Borsboom, D. (2017). A network theory of mental disorders. World Psychiatry, 16(1), 5-13. https:// doi.org/10.1002/wps.20375

Borsboom, D., \& Cramer, A. O. J. (2013). Network Analysis: An Integrative Approach to the Structure of Psychopathology. Annual Review of Clinical Psychology, 9(1), 91-121. https://doi.org/10.1146/annurev-clinpsy-050212-185608

Bradley, K. A., DeBenedetti, A. F., Volk, R. J., Williams, E. C., Frank, D., \& Kivlahan, D. R. (2007). AUDIT-C as a Brief Screen for Alcohol Misuse in Primary Care. Alcoholism: Clinical and Experimental Research, 31(7), 1208-1217. https://doi.org/10.1111/j.15300277.2007.00403.x

David, S. J., Marshall, A. J., Evanovich, E. K., \& Mumma, G. H. (2018). Intraindividual Dynamic Network Analysis - Implications for Clinical Assessment. Journal of Psychopathology and Behavioral Assessment, 40(2), 235-248. https://doi.org/10.1007/s10862-017-9632-8

Epskamp, S., Borsboom, D., \& Fried, E. I. (2018). Estimating psychological networks and their accuracy: A tutorial paper. Behavior Research Methods, 50(1), 195-212. https://doi.org/10.3758/s13428-017-0862-1

Fisher, A. J., Reeves, J. W., Lawyer, G., Medaglia, J. D., \& Rubel, J. A. (2017). Exploring the idiographic dynamics of mood and anxiety via network analysis. Journal of Abnormal Psychology, 126(8), 1044-1056. https://doi.org/10.1037/abn0000311

Fisher, A. J., Bosley, H. G., Fernandez, K. C., Reeves, J. W., Soyster, P. D., Diamond, A. E., \& Barkin, J. (2019). Open trial of a personalized modular treatment for mood and anxiety. Behaviour Research and Therapy, 116, 69-79. https://doi.org/10.1016/j.brat.2019.01.010 
Frank, A., Fatke, B., Frank, W., Förstl, H., \& Hölzle, P. (2020). Depression, Dependence and Prices of the COVID-19-Crisis. Brain, Behavior, and Immunity. https://doi.org/10.1016/j.bbi.2020.04.068

Hao, F., Tan, W., Jiang, L., Zhang, L., Zhao, X., Zou, Y., Hu, Y., Luo, X., Jiang, X., McIntyre, R. S., Tran, B., Sun, J., Zhang, Z., Ho, R., Ho, C., \& Tam, W. (2020). Do psychiatric patients experience more psychiatric symptoms during COVID-19 pandemic and lockdown? A casecontrol study with service and research implications for immunopsychiatry. Brain, Behavior, and Immunity. https://doi.org/10.1016/j.bbi.2020.04.069

Holmes, E. A., O’Connor, R. C., Perry, V. H., Tracey, I., Wessely, S., Arseneault, L., Ballard, C., Christensen, H., Silver, R. C., Everall, I., Ford, T., John, A., Kabir, T., King, K., Madan, I., Michie, S., Przybylski, A. K., Shafran, R., Sweeney, A., ... Bullmore, E. (2020). Multidisciplinary research priorities for the COVID-19 pandemic: a call for action for mental health science. The Lancet Psychiatry, 7(6), 547-560. https://doi.org/10.1016/S22150366(20)30168-1

Maj, M., Stein, D. J., Parker, G., Zimmerman, M., Fava, G. A., De Hert, M., Demyttenaere, K., McIntyre, R. S., Widiger, T., \& Wittchen, H. (2020). The clinical characterization of the adult patient with depression aimed at personalization of management. World Psychiatry, 19(3), 269-293. https://doi.org/10.1002/wps.20771

Morin, Belleville, G., Bélanger, L., \& Ivers, H. (2011). The Insomnia Severity Index: psychometric indicators to detect insomnia cases and evaluate treatment response. Sleep, 34(5), 601-608.

Morin, Moullec, G., Maïano, C., Layet, L., Just, J.-L., \& Ninot, G. (2011). Psychometric properties of the Center for Epidemiologic Studies Depression Scale (CES-D) in French clinical and nonclinical adults. Revue d'Épidémiologie et de Santé Publique, 59(5), 327-340. https://doi.org/10.1016/j.respe.2011.03.061

Moullec, G., Maïano, C., Morin, A. J. S., Monthuy-Blanc, J., Rosello, L., \& Ninot, G. (2011). A very short visual analog form of the Center for Epidemiologic Studies Depression Scale (CES-D) for the idiographic measurement of depression. Journal of Affective Disorders, 128(3), 220-234. https://doi.org/10.1016/j.jad.2010.06.006 
Muntingh, A. D., van der Feltz-Cornelis, C. M., van Marwijk, H. W., Spinhoven, P., Penninx, B. W., \& van Balkom, A. J. (2011). Is the beck anxiety inventory a good tool to assess the severity of anxiety? A primary care study in The Netherlands study of depression and anxiety (NESDA). BMC Family Practice, 12, 66. https://doi.org/10.1186/1471-2296-12-66

Pfefferbaum, B., \& North, C. S. (2020). Mental Health and the Covid-19 Pandemic. New England Journal of Medicine, 383(6), 510-512. https://doi.org/10.1056/NEJMp2008017

Pinkham, A. E., Ackerman, R. A., Depp, C. A., Harvey, P. D., \& Moore, R. C. (2020). A Longitudinal Investigation of the Effects of the COVID-19 Pandemic on the Mental Health of Individuals with Pre-existing Severe Mental Illnesses. Psychiatry Research, 294, 113493. https://doi.org/10.1016/j.psychres.2020.113493

Roth, T., Seiden, D., Sainati, S., Wang-Weigand, S., Zhang, J., \& Zee, P. (2006). Effects of ramelteon on patient-reported sleep latency in older adults with chronic insomnia. Sleep Medicine, 7(4), 312-318. https://doi.org/10.1016/j.sleep.2006.01.003

Snippe, E., Viechtbauer, W., Geschwind, N., Klippel, A., de Jonge, P., \& Wichers, M. (2017). The Impact of Treatments for Depression on the Dynamic Network Structure of Mental States: Two Randomized Controlled Trials. Scientific Reports, 7(1). https://doi.org/10.1038/srep46523

Taquet, M., Quoidbach, J., Fried, E. I., \& Goodwin, G. M. (2021). Mood Homeostasis Before and During the Coronavirus Disease 2019 (COVID-19) Lockdown Among Students in the Netherlands. JAMA Psychiatry, 78(1), 110. https://doi.org/10.1001/jamapsychiatry.2020.2389

van Hees, V. T., Sabia, S., Jones, S. E., Wood, A. R., Anderson, K. N., Kivimäki, M., Frayling, T. M., Pack, A. I., Bucan, M., Trenell, M. I., Mazzotti, D. R., Gehrman, P. R., Singh-Manoux, B. A., \& Weedon, M. N. (2018). Estimating sleep parameters using an accelerometer without sleep diary. Scientific Reports, 8(1), 12975. https://doi.org/10.1038/s41598-018$31266-\mathrm{z}$ 
Västfjäll, D., \& Gärling, T. (2007). Validation of a Swedish short self-report measure of core affect. Scandinavian Journal of Psychology, 48(3), 233-238. https://doi.org/10.1111/j.14679450.2007.00595.x

Wichers, Groot, P. C., \& Psychosystems, ESM Group, EWS Group. (2016). Critical Slowing Down as a Personalized Early Warning Signal for Depression. Psychotherapy and Psychosomatics, 85(2), 114-116. https://doi.org/10.1159/000441458

Wichers, M. (2014). The dynamic nature of depression: a new micro-level perspective of mental disorder that meets current challenges. Psychological Medicine, 44(07), 1349-1360. https:// doi.org/10.1017/S0033291713001979

Wood, S. N. (2017). Generalized Additive Models: An Introduction with R, Second Edition (2 edition). Chapman and Hall/CRC. 
Table 1

\begin{tabular}{lll}
\hline Depressive symptoms & Item & References \\
\hline Appetite loss* & My appetite is good & (Moullec et al., 2011) \\
Sleep quality* & During last night, my sleep quality(Fanning et al., 2016) \\
& was good & \\
Psychomotor retardation & I feel as if I'm slowed down & (Vansimaeys et al., 2017) \\
Fatigue & I am tired & (Rintala et al., 2020) \\
Guilty & I am feeling guilty & (Brenner \& Ben-Zeev, 2014) \\
Concentration & I have trouble keeping my mind on(Moullec et al., 2011) \\
& what I am doing & \\
Core affects (I am feeling) & Bipolar adjectives scales & (VäStfjäLl \& GäRling, 2007) \\
Valence 1* & Sad-glad & \\
Valence 2* & Depressed-happy & \\
Valence 3* & Displeased-pleased & \\
Arousal 1* & Dull-peppy & \\
Arousal 2* & Passive-active & \\
Arousal 3* & Sleepy-awake & \\
\hline
\end{tabular}

$*=$ inverted item

Table 2

Descriptive findings for case 1 and 2

\begin{tabular}{|c|c|c|}
\hline & Case 1 & Case 2 \\
\hline Age & 36 & 20 \\
\hline Marital status & Single & Couple \\
\hline Child & No & No \\
\hline Income & $40,000-60,000 \mathrm{ca} \$$ & $<20,000 \mathrm{ca} \$$ \\
\hline Smoking & No & No \\
\hline $\begin{array}{l}\text { Alcohol Use Disorders Identification } \\
\text { Test (short form) }\end{array}$ & 3 & 1 \\
\hline Insomnia Severity Index & 22 & 14 \\
\hline $\begin{array}{l}\text { Center for Epidemiologic Studies } \\
\text { Depression }\end{array}$ & 39 & 34 \\
\hline Beck Anxiety Inventory & 46 & 44 \\
\hline Sleep Efficiency & $\mathrm{M}=85.5 \%$ & $\mathrm{M}=84.3 \%$ \\
\hline Total Sleep Time & $\mathrm{M}=5.2$ hours & $\mathrm{M}=7.7$ hours \\
\hline Adherence rate to EMA & $86 / 90$ time points & $79 / 90$ time points \\
\hline Adherence rate to accelerometer & 29/30 nights & 29/30 nights \\
\hline
\end{tabular}

Notes. EMA = Ecological Momentary Assessment 
Figure 1

Case 1: Smoothed time series of self-reported affects

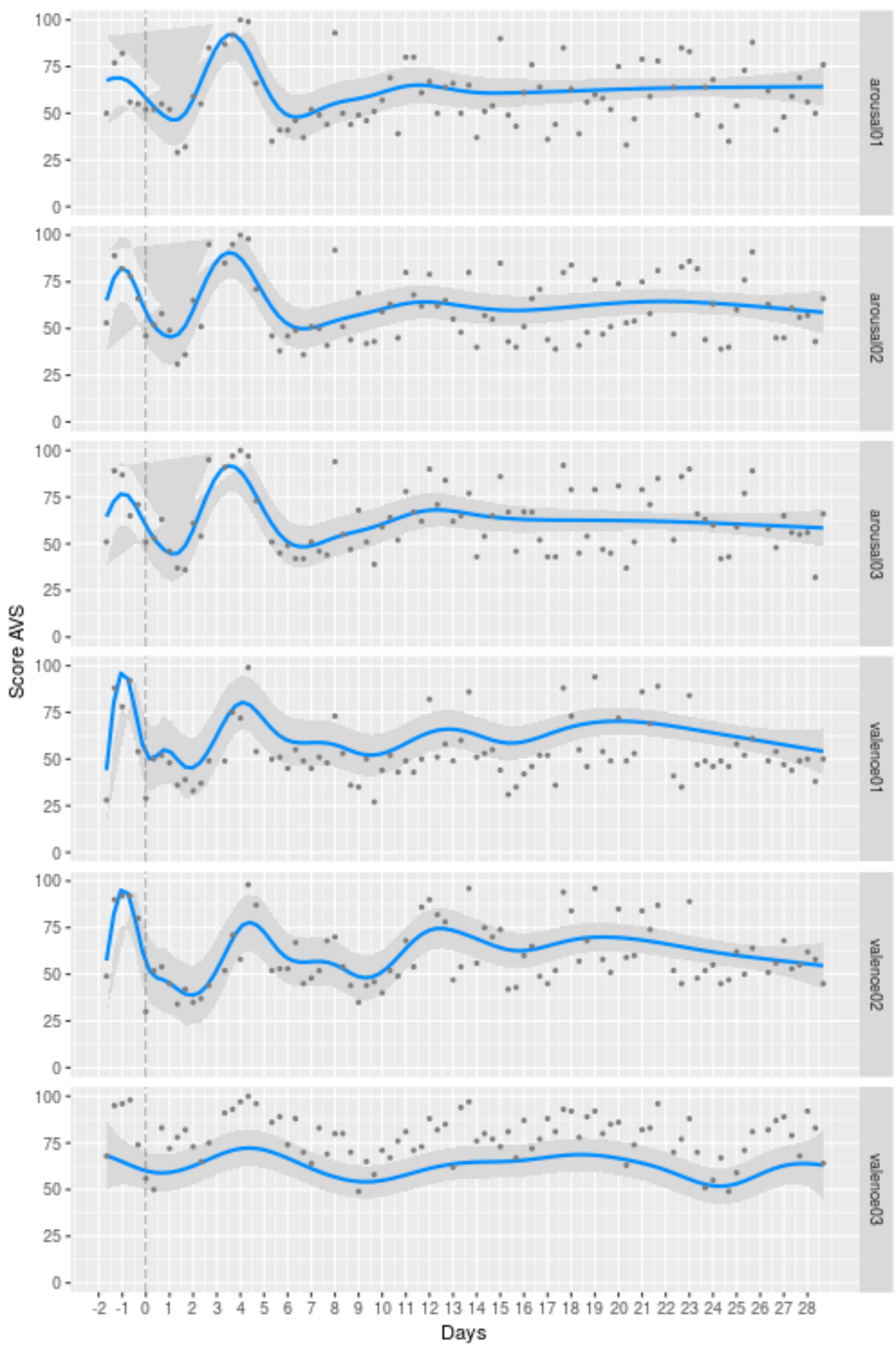

Notes. The dotted line represents the lockdown begins after the inclusion of participants. 
Figure 2

Contemporaneous and temporal $(t+1)$ networks for case 1 and 2
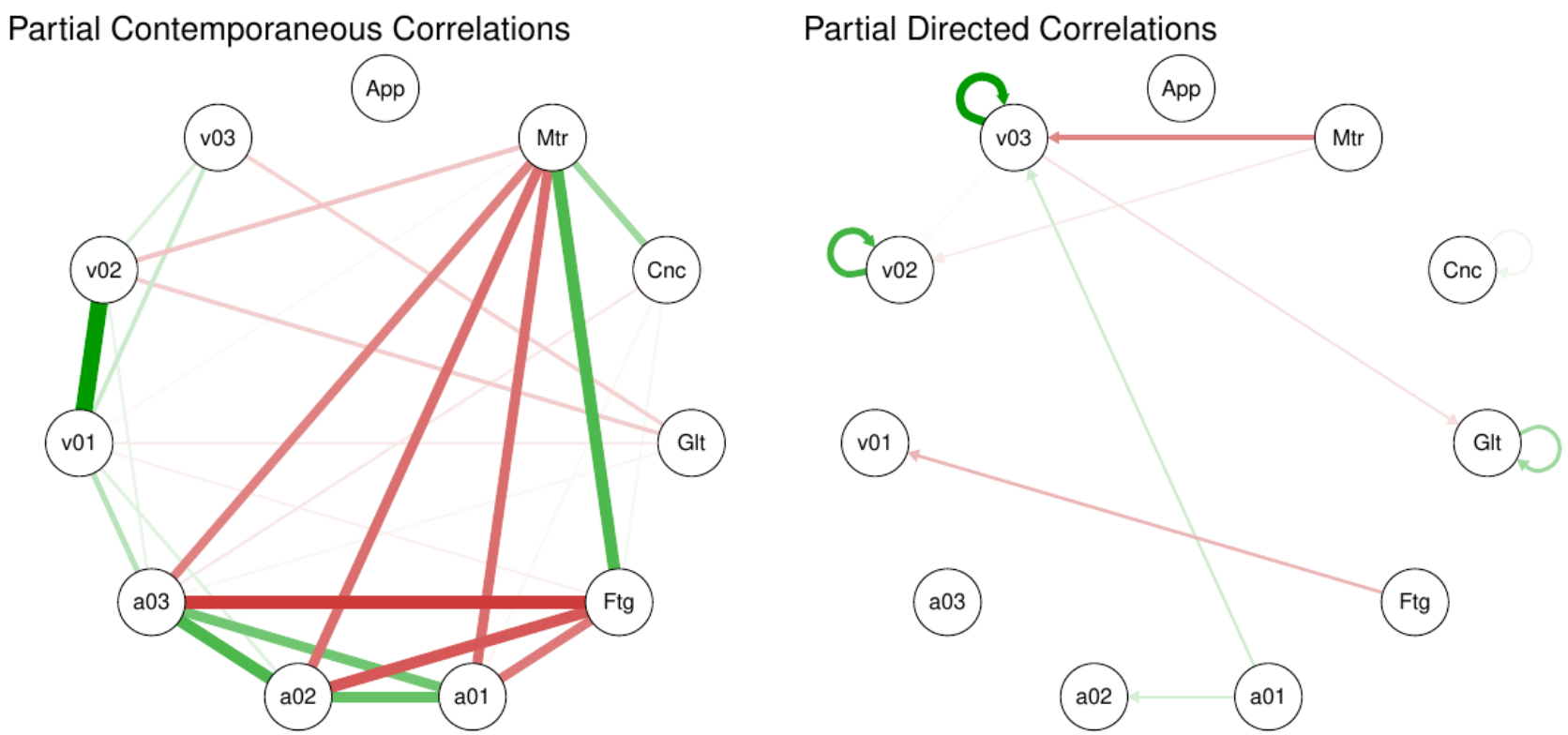

Notes. App $=$ Appetite loss, Mtr $=$ Psychomotor retardation, Cnc $=$ Concentration, Glt $=$ Guilty, Ftg $=$ Fatigue, a01 $=\mathrm{I}$ am feeling [Dull - peppy] , a02 $=\mathrm{I}$ am feeling [Passive - active], a03 $=\mathrm{I}$ am feeling [Sleepy awake], $\mathrm{v} 01=\mathrm{I}$ am feeling [Sad - glad], v02 = I am feeling [Depresed - happy], v03 = I am feeling [Displeased- pleased]

Figure 3

Contemporaneous and temporal $(t+1)$ networks for case 2

Partial Contemporaneous Correlations

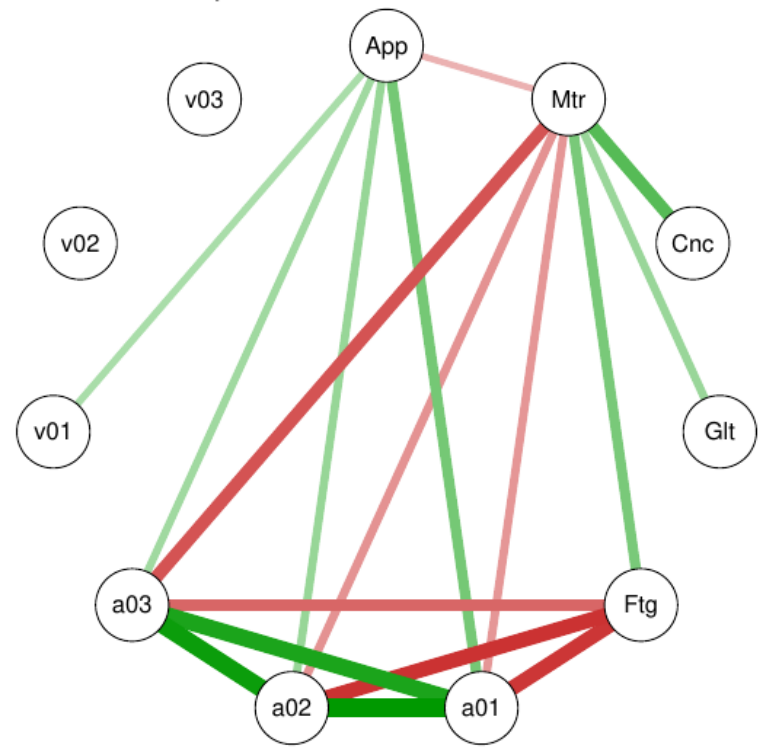

Partial Directed Correlations

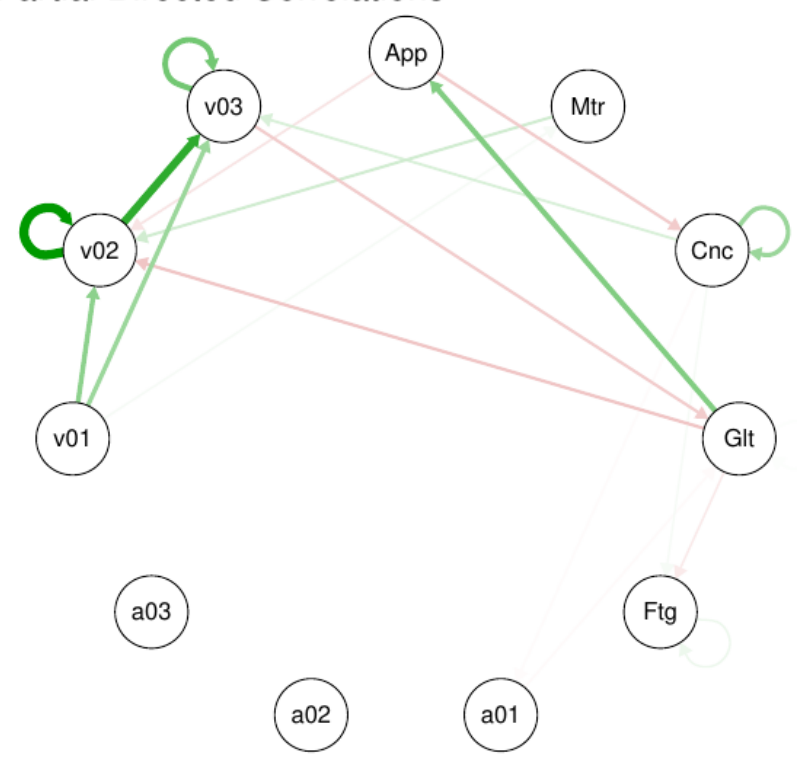

Notes. App $=$ Appetite loss, Mtr $=$ Psychomotor retardation, $\mathrm{Cnc}=$ Concentration, Glt $=$ Guilty, Ftg $=$ Fatigue, a01 $=\mathrm{I}$ am feeling [Dull - peppy] , a02 $=\mathrm{I}$ am feeling [Passive - active], a03 $=\mathrm{I}$ am feeling [Sleepy awake], $\mathrm{v} 01=\mathrm{I}$ am feeling [Sad - glad], v02 = I am feeling [Depresed - happy], v03 = I am feeling [Displeased- pleased] 


\section{Declaration of interests}

The authors declare no competing interests.

\section{Funding}

PB is supported by Université du Québec à Montréal, Institut Universitaire en Santé Mentale de Montréal and by a salary award from the Fonds de recherche du Québec - Santé.

\section{Acknowledgment}

The authors would like to T.Kaiser. Without the generosity of our participants, the project could not have taken place. 